\title{
Índices de gravidade em vítimas de trauma contuso na terapia intensiva: capacidade preditiva de mortalidade*
}

\author{
Severity indexes of blunt trauma victims in intensive therapy: prediction capacity for mortality \\ Índices de gravedad en las víctimas de traumas contusos en los cuidados intensivos: capacidad \\ predictiva de la mortalidad
}

Como citar este artigo:

Lima KP, Nogueira LS, Barbosa G, Bonfim AKS, Sousa RMC. Severity indexes of blunt trauma victims in intensive therapy: prediction capacity for mortality. Rev Esc Enferm USP. 2021;55:e03747. https://doi.org/10.1590/S1980-220X2020003203747

\section{Kézia Porto Lima ${ }^{1,2}$ \\ Lilia de Souza Nogueira ${ }^{1}$ \\ Genesis Barbosa ${ }^{1,3}$ \\ Ane Karoline Silva Bonfim ${ }^{1}$ \\ Regina Marcia Cardoso de Sousa ${ }^{1}$}

* Extraído da dissertação: "Índices de gravidade em vítimas de trauma internadas em Unidade de Terapia Intensiva", Programa de Pós-Graduacão em Enfermagem na Saúde do Adulto, Universidade de São Paulo, 2018.

${ }^{1}$ Universidade de São Paulo, Escola de Enfermagem, Programa de Pós-Graduação em Enfermagem na Saúde do Adulto, São Paulo, SP, Brasil.

${ }^{2}$ Faculdade dos Carajás, Marabá, PA, Brasil.

${ }^{3}$ Universidade Federal do Rio de Janeiro, Campus Professor Aloísio Teixeira, Macaé, RJ, Brasil.

\begin{abstract}
Objective: To identify the predictive capacity for mortality of the indexes Revised Trauma Score, Rapid Emergency Medicine Score, modified Rapid Emergency Medicine Score, and Simplified Acute Physiology Score III in blunt trauma victims hospitalized in an intensive care unit and compare their performance. Method: Retrospective cohort of patients with blunt trauma in an intensive care unit from medical records. Receiver Operating Characteristic and a 95\% confidence interval of the area under the curve were analyzed to compare results. Results: Out of 165 analyzed patients, $66.7 \%$ have received surgical treatment. The mortality in the intensive care unit and in the hospital was $17.6 \%$ and $20.6 \%$, respectively. For the mortality in intensive therapy, the area under the curve varied from 0.672 to 0.738 ; however, better results have been observed in surgical patients ( 0.747 to 0.811 ). Similar results have been observed for hospital mortality. In all analyses, the areas under the curve of the indexes presented no significant difference. Conclusion: The accuracy of the severity indexes was moderate, with an improved performance when applied to surgical patients. The four indexes presented a similar prediction for the analyzed outcomes.
\end{abstract}

\section{DESCRIPTORS}

Wounds and Injuries; Severity of Illness Index; Trauma Severity Indexes; Mortality; Prognosis; ROC Curve. 


\section{INTRODUÇÃO}

Trauma é um importante problema de saúde pública no Brasil e no mundo pela elevada taxa de morte ou graves consequências que geram incapacidades temporárias ou permanentes nas vítimas. Segundo informações do Departamento de Informática do Sistema Único de Saúde do Brasil (DATASUS), em 2008, ocorreram 135.936 óbitos por causa externa no Brasil; em 2017, a mortalidade por essa ocorrência foi de $158.657 \operatorname{casos}^{(1)}$, totalizando um aumento de $17,0 \%$ em apenas uma década, ao passo que o crescimento populacional no período foi de $9,5 \% \%^{(2)}$.

Dados do DATASUS dos últimos 20 anos ainda apontam as causas externas como as principais responsáveis pelos óbitos de indivíduos na faixa etária de 1 a 49 anos; em 2017, $72,1 \%$ das mortes de indivíduos entre 15 e 29 anos de idade ocorreram por trauma ${ }^{(1)}$.

A garantia do melhor atendimento à vítima de trauma depende da eficiência de todos os profissionais envolvidos em seu cuidado, desde o atendimento na cena do trauma até o completo tratamento em ambiente intra-hospitalar, que será concluído com a reabilitação e reinserção desse indivíduo na sociedade ${ }^{(3)}$. É necessário que os profissionais envolvidos em todas essas etapas tenham conhecimento da gravidade do quadro clínico do traumatizado para assumir condutas imediatas de intervenção e triagem, planejar a assistência e qualificar os serviços envolvidos no atendimento ${ }^{(4-5)}$.

Para atender esse propósito, foram desenvolvidos os escores de mortalidade e de prognóstico, comumente chamados de índices de gravidade ${ }^{(6-7)}$. São muitos os indicadores de gravidade que podem ser utilizados para as vítimas de trauma: os específicos para esse tipo de ocorrência, os elaborados para indivíduos atendidos em serviços de emergência e, nos casos graves e encaminhados para Unidade de Terapia Intensiva (UTI), os índices próprios para os pacientes dessas unidades.

Vítimas de trauma são frequentes em UTI e o risco de morrer as qualifica como um grupo cuja gravidade clínica é de grande interesse. Há, no entanto, o desafio da escolha de um instrumento adequado para esse fim. Na literatura, o Revised Trauma Score (RTS) $)^{(8)}$, o Rapid Emergency Medicine Score $(\mathrm{REMS})^{(9)}$, o modified Rapid Emergency Medicine Score (mREMS) ${ }^{(10)}$ e o Simplified Acute Physiology Score III (SAPS III) ${ }^{(11)}$ têm se destacado como indicadores de base fisiológica para estimar a gravidade de pacientes admitidos em serviços de emergência e na UTI.

Entre os mais utilizados índices de gravidade específicos de trauma, observa-se o índice prognóstico RTS, desenvolvido em 1989, cujos coeficientes são provenientes de análise de regressão logística aplicada na ampla base de dados Major Trauma Outcome Study ${ }^{(8)}$.

O REMS é um indicador de gravidade fisiológico utilizado na emergência derivado do Acute Physiologic and Chronic Health Evaluation II (APACHE II), o qual requer uma avaliação rápida para obtenção de alguns parâmetros fisiológicos e permite cálculo imediato, sem uso de exames laboratoriais ou complementares ${ }^{(9)}$.

Ainda que estudos tenham considerado o uso do REMS adequado para vítimas de trauma, pesquisadores identificaram que o escore deveria ser ajustado para melhor predizer a mortalidade desses pacientes. Foi desenvolvido então o mREMS, publicado em $2017^{(10)}$.

$\mathrm{Na}$ validação dessa nova versão do índice, pesquisadores identificaram que o mREMS forneceu previsões precisas para a mortalidade intra-hospitalar, superando o Injury Severity Score (ISS) e o Shock Index (SI) e igualando-se ao RTS e Mechanism, Glasgow Coma Score, Age, and Arterial Pressure (MGAP). Em consequência, o REMS tem sido considerado uma ferramenta simples, objetiva e de valor para vítimas de trauma no cenário de emergência ${ }^{(10)}$.

Para avaliar a gravidade de pacientes de UTI, tem-se aplicado o índice SAPS III ${ }^{(11)}$, um sistema uniformizado e aceito internacionalmente para essa finalidade, resultado do aprimoramento do APACHE e de versões do SAPS.

$\mathrm{O}$ uso dos índices de gravidade, juntamente com os registros do evento traumático, representa diversas possibilidades de aplicação clínica e científica para melhor descrever e classificar as vítimas de trauma. Nesse sentido, o aperfeiçoamento do uso dos indicadores para mensurar a gravidade das vítimas de trauma é um trabalho em desenvolvimento contínuo ${ }^{(4,7)}$. Há uma grande variedade de índices de gravidade que podem ser utilizados na assistência às vítimas de trauma nos diferentes momentos do seu cuidado; porém, é importante identificar aqueles que oferecem uma adequada precisão.

Diante dos aspectos apresentados, este estudo tem como objetivos identificar a capacidade preditiva de mortalidade, na UTI e no ambiente hospitalar, do RTS, REMS, mREMS e SAPS III em vítimas de trauma contuso internadas nessa unidade e comparar seu desempenho.

\section{MÉTODO}

\section{TIPO DE ESTUDO}

Trata-se de um estudo tipo coorte retrospectiva, que computou informações dos prontuários de vítimas de trauma desde a admissão na emergência até a alta hospitalar.

\section{Cenário}

O estudo foi realizado em uma UTI referenciada, composta de 24 leitos, especializada em Emergências Cirúrgicas e Trauma, que presta atendimento aos casos de trauma de alta complexidade no estado de São Paulo, Brasil.

\section{Critérios de SELEÇão}

Fizeram parte da casuística todos os pacientes que atenderam os seguintes critérios de elegibilidade: vítimas de trauma contuso, idade igual ou superior a 18 anos e admitidos na UTI no período de $1^{\circ}$ de agosto de 2014 a 31 de julho de 2016. Traumas contusos e penetrantes têm diferentes etiologias, manifestações clínicas, tratamentos e mortalidade, circunstâncias que indicam análises distintas para esses tipos de trauma. Durante a coleta de dados, $90 \%$ dos atendidos no local deste estudo apresentaram trauma contuso. Em consequência, optou-se por analisar os índices em vítimas desse tipo de lesão.

Foram excluídos dessa amostra indivíduos que chegaram ao pronto-socorro 24 horas após a ocorrência do evento traumático, vítimas de enforcamento, sufocamento, afogamento 
ou quase afogamento, envenenamento, queimadura e eletrocussão. A exclusão dos que chegaram ao pronto-socorro 24 horas após a ocorrência do evento traumático foi estabelecida tendo em vista que as condições clínicas iniciais das vítimas de trauma são utilizadas para cálculo dos índices e apontadas como importantes indicadores de mortalidade ou sobrevida. As vítimas das causas externas elencadas foram excluídas, considerando as importantes especificidades da fisiopatologia desses traumas perante os demais.

\section{Coleta de dados}

A partir de consulta ao livro de admissões dos pacientes na UTI do estudo, foi elaborada uma lista de doentes que atenderam aos critérios de elegibilidade da pesquisa. Com base nessa lista, solicitou-se ao Serviço de Arquivo Médico e Estatístico da instituição a localização dos prontuários dos pacientes para consulta e compilação dos dados de interesse para a pesquisa.

A análise dos prontuários permitiu verificar se os pacientes atendiam aos critérios de elegibilidade, bem como o preenchimento de duas fichas utilizadas na coleta de dados. Os dados incluídos no primeiro instrumento permitiram o cálculo do RTS, REMS e mREMS a partir de registros da admissão da vítima no departamento de emergência, a identificação dos pacientes cirúrgicos e não cirúrgicos, mortos e sobreviventes durante a internação hospitalar, além da caracterização da amostra (sexo, idade, causa externa, tipo de suporte pré-hospitalar e tempo de internação hospitalar). O SAPS III, índice de gravidade aplicado e registrado regularmente na UTI pela equipe médica, teve seu valor transcrito para o segundo instrumento de coleta, que também contemplou informações sobre a internação na terapia intensiva até a alta hospitalar.

Tendo em conta que os índices de gravidade analisados foram elaborados para previsão de mortalidade em UTI ${ }^{(11)}$ ou hospitalar ${ }^{(8-10)}$, foram realizadas análises considerando-se como variável dependente mortalidade na UTI e durante toda a internação hospitalar.

O RTS, REMS, mREMS e SAPS III foram as variáveis independentes desta investigação. O RTS é um índice cujo cálculo baseia-se no escore da Escala de Coma de Glasgow (ECGl), valor da pressão arterial sistólica (PAS) e frequência respiratória (FR). Para estimar a probabilidade de sobrevida do traumatizado, são atribuídos valores de zero a 4 a cada parâmetro fisiológico mensurado na admissão hospitalar que, posteriormente, são multiplicados pelos seus respectivos pesos (0,9368 para ECG1, 0,7326 para PAS e 0,2908 para FR) e somados $^{(8)}$. O RTS pode variar de zero a 7,8408 e quanto maior o valor final, melhor é o prognóstico da vítima. A probabilidade de sobrevida das vítimas de trauma apresenta correspondência com a pontuação do RTS, conforme proposto pelos autores do índice ${ }^{(8)}$.

O REMS é composto por ECGl, frequência cardíaca (FC), pressão arterial média (PAM), FR, saturação de oxigênio (SatO2) e idade. Conforme os valores observados na admissão no serviço de emergência, essas variáveis recebem pontuação de zero a quatro pontos, exceto a idade, que tem variação de zero a seis; o escore total do REMS é a somatória da pontuação obtida nessas variáveis ${ }^{(9)}$. O cálculo do mREMS inclui idade, PAS, FC, FR, SatO2 e ECG1. Aos valores observados nesses parâmetros, são atribuídas pontuações de 0 a 4 , com exceção da ECGl, que apresenta pontuação de 0 a 6; o valor do mREMS é a soma dessas pontuações ${ }^{(10)}$. Os escores do REMS e mREMS variam de zero a 26 e as maiores pontuações indicam maior risco de morrer ${ }^{(9-10)}$.

$\mathrm{Na}$ aplicação do SAPS III, são utilizados os valores dos três grupos de variáveis: idade e informações sobre o estado prévio de saúde (comorbidades, tempo de permanência hospitalar antes da admissão na UTI, procedência e uso de drogas vasoativas); circunstâncias da admissão na UTI (razões da internação na UTI, sítio anatômico da cirurgia, se aplicável, tipo de admissão na UTI - programada ou não programada, status cirúrgico e presença de infecção nosocomial e/ou respiratória) e variáveis fisiológicas (temperatura, PAS, FC, oxigenação, pH arterial, creatinina, bilirrubina, hematócrito, leucócitos, plaquetas e ECGl). Para pontuar esse índice, são considerados os piores valores atribuídos às variáveis fisiológicas na primeira hora de internação do paciente em UTI ${ }^{(11)}$. Cada item do SAPS III tem sua pontuação específica e o escore final é a somatória desses valores. O menor escore que pode ser atribuído ao índice é 16 e o maior é 217 pontos; quanto maior a pontuação, mais grave é o estado do paciente. Quando essa pontuação é convertida por equação da regressão logística, o índice mostra a probabilidade de mortalidade hospitalar ${ }^{(4,11)}$.

\section{ANÁlise E TRATAMENTO DOS DADOS}

O banco de dados computadorizado deste estudo foi construído com uso do software Statistical Package for the Social Sciences (SPSS) versão 22, que foi utilizado nas provas estatísticas, conforme orientação de especialista na área. Exceto o SAPS III, que foi transcrito dos prontuários, os demais índices foram calculados em planilha eletrônica.

Análises inferenciais foram realizadas para avaliar o desempenho dos índices de gravidade (RTS, REMS, mREMS e SAPS III) e comparar sua capacidade preditiva para a ocorrência de óbito das vítimas durante a internação na UTI e no hospital, considerando a casuística total e os pacientes submetidos ou não a tratamento cirúrgico separadamente. A prova diagnóstica Receiver Operating Characteristic (curva ROC) foi utilizada para análise do desempenho dos índices. O ponto de corte foi identificado pelo índice de Youden e calcularam-se os valores de sensibilidade, especificidade, valor preditivo positivo (VPP) e valor preditivo negativo (VPN). A diferença no desempenho dos índices foi identificada pela análise da área sob a curva (AUC) e intervalo de confiança (IC) de 95\%.

\section{Aspectos ÉTICOS}

O estudo foi aprovado pelo Comitê de Ética em Pesquisa da instituição (protocolo no 2.490.677). Todos os dados dos pacientes foram salvos e protegidos em computador, com acesso restrito apenas aos pesquisadores deste estudo, garantindo a segurança e o anonimato das informações coletadas. 


\section{RESULTADOS}

A população do estudo constituiu-se de 165 vítimas de trauma contuso admitidas na UTI com idade média de 38,5 e desvio padrão (dp) de 15,4 anos. Quanto ao sexo, predominou o masculino, representando $81,2 \%$ do total da amostra. A causa externa de maior ocorrência foi o acidente motociclístico $(33,3 \%)$, seguido por atropelamento $(27,3 \%)$ e queda (20,6\%). Em 43,0\% dos casos, as vítimas chegaram à unidade de emergência por suporte básico de vida; porém, mais de metade das vítimas $(53,4 \%)$ foi atendida por suporte avançado de vida aéreo $(35,8 \%)$ ou terrestre $(17,6 \%)$.

Da amostra analisada, 110 pacientes $(66,7 \%)$ foram submetidos a tratamento cirúrgico. A média de tempo de internação dos pacientes na UTI foi de 16,8 dias $(\mathrm{dp}=33,4)$ e, no hospital, de 24,6 dias $(\mathrm{dp}=40,6)$. Um total de 29 pacientes $(17,6 \%)$ morreu na terapia intensiva e a taxa de mortalidade hospitalar foi de 20,6\%; cinco pacientes morreram no hospital após a alta da UTI.

A probabilidade de sobrevida estimada pelo RTS na casuística variou de $98,8 \%$ a $2,7 \%$ e a sobrevida inferior a $50 \%$ foi estimada em 18,7\%. A média do REMS foi de 4,8 $(\mathrm{dp}=3,42)$; pontuações $\geq 6 \mathrm{e} \leq 13$ foram observadas em $35,7 \%$ da casuística e $>13$ em 1,2\%. A média do mREMS foi de $5,1(\mathrm{dp}=3,7)$ e $41,2 \%$ das vítimas apresentaram pontuações $\geq 6$ e $\leq 13$, ao passo que $1,8 \%$ obtiveram pontuação $>13$. A média do SAPS III foi de $48,6(\mathrm{dp}=17,1)$ e a maioria das vítimas obteve escore entre 32 e 67.

As AUC/ROC para mortalidade em UTI das vítimas de trauma contuso foram próximas a 0,70 para todos os índices analisados (variação de 0,672 a 0,738), conforme se observa na Figura 1.

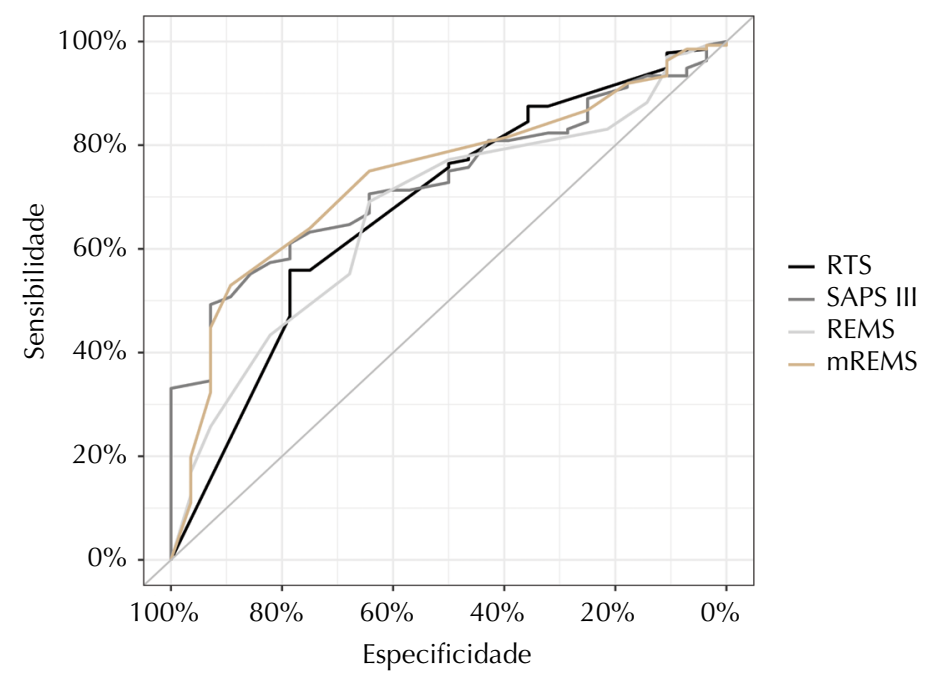

ROC - Receiver Operating Characteristic; RTS - Revised Trauma Score; SAPS III - Simplified Acute Physiology Score III; REMS - Rapid Emergency Medicine Score; mREMS - modified Rapid Emergency Medicine Score; UTI - Unidade de Terapia Intensiva ( $n=165)$.

Figura 1 - Curvas ROC dos índices RTS, SAPS III, REMS e mREMS para discriminar, nas vítimas de trauma, os mortos e sobreviventes à alta da UTI - São Paulo, SP, Brasil, 2014-2016.

Os resultados da Tabela 1 mostram que as AUC/ ROC para mortalidade em UTI foram similares à mortalidade hospitalar em todos os índices, segundo os valores dos IC95\% observados. Além disso, os IC95\% indicaram, na comparação do desempenho dos índices, que não houve diferença dos resultados das AUC/ROC no nível de 0,05, na medida em que apresentaram valores sobrepostos, conforme resultados da Tabela 1 .
O SAPS III apresentou maior AUC que os demais índices quando analisada a mortalidade hospitalar e foi similar ao mREMS para mortalidade em UTI. Porém, o SAPS III apresentou menor sensibilidade e VPN do que os demais índices para os dois desfechos analisados.

Tabela 1 - Capacidade preditiva do RTS, REMS, mREMS e SAPS III para mortalidade em UTI e hospitalar - São Paulo, SP, Brasil, $2014-2016$.

\begin{tabular}{lcccc}
\hline \multirow{2}{*}{ Métricas da ROC } & \multicolumn{4}{c}{ Mortalidade em UTI } \\
\cline { 2 - 5 } & RTS & REMS & mREMS & SAPS III \\
\hline AUC/ROC & 0,682 & 0,672 & 0,738 & 0,731 \\
IC95\% & $0,574-0,789$ & $0,569-0,775$ & $0,646-0,831$ & $0,646-0,815$ \\
\hline
\end{tabular}


...continuação

\begin{tabular}{|c|c|c|c|c|}
\hline \multirow{2}{*}{ Métricas da ROC } & \multicolumn{4}{|c|}{ Mortalidade em UTI } \\
\hline & RTS & REMS & mREMS & SAPS III \\
\hline Ponto de corte & 6,2 & 5,5 & 4,5 & 44,5 \\
\hline Sensibilidade & $78,6 \%$ & $69,1 \%$ & $52,9 \%$ & $49,3 \%$ \\
\hline Especificidade & $55,9 \%$ & $64,3 \%$ & $89,3 \%$ & $92,9 \%$ \\
\hline VPP & $26,8 \%$ & $90,4 \%$ & $96,0 \%$ & $97,1 \%$ \\
\hline VPN & $92,7 \%$ & $30,0 \%$ & $28,1 \%$ & $27,4 \%$ \\
\hline \multirow{2}{*}{ Métricas da ROC } & \multicolumn{4}{|c|}{ Mortalidade hospitalar } \\
\hline & RTS & REMS & mREMS & SAPS III \\
\hline $\mathrm{AUC} / \mathrm{ROC}$ & 0,646 & 0,637 & 0,688 & 0,734 \\
\hline IC95\% & $0,541-0,752$ & $0,535-0,738$ & $0,589-0,787$ & $0,652-0,816$ \\
\hline Ponto de corte & 6,2 & 5,5 & 6,5 & 44,5 \\
\hline Sensibilidade & $72,7 \%$ & $68,7 \%$ & $74,8 \%$ & $50,4 \%$ \\
\hline Especificidade & $55,7 \%$ & $57,6 \%$ & $57,6 \%$ & $90,9 \%$ \\
\hline VPP & $29,3 \%$ & $86,5 \%$ & $87,5 \%$ & $95,6 \%$ \\
\hline VPN & $89,0 \%$ & $31,7 \%$ & $36,5 \%$ & $31,6 \%$ \\
\hline
\end{tabular}

AUC/ROC - Area Under Curve/Receiver Operating Characteristic; RTS - Revised Trauma Score; REMS - Rapid Emergency Medicine Score; mREMS - modified Rapid Emergency Medicine Score; SAPS III - Simplified Acute Physiology Score III; UTI - Unidade de Terapia Intensiva; IC - Intervalo de Confiança; VPP - Valor Preditivo Positivo; VPN - Valor Preditivo Negativo $(n=165)$.

A Tabela 2 mostra que, para pacientes cirúrgicos, RTS, REMS e mREMS apresentaram maior AUC/ROC quando analisada a mortalidade em UTI; porém, o SAPS III apresentou valor similar para mortalidade em UTI e hospitalar. $\mathrm{Na}$ análise do IC95\%, os demais índices, RTS, REMS e mREMS, também apresentaram resultados que não diferiram significativamente entre a condição de saída da UTI e do hospital.

Os dados da Tabela 2, juntamente com as curvas ROC dos índices apresentados na Figura 2, mostram AUC entre
0,747 e 0,811 na predição de mortalidade na UTI das vítimas de trauma submetidas a tratamento cirúrgico. O SAPS III e o mREMS apresentaram as mais altas AUC, com valores superiores a 0,80 ; entretanto, os menores valores de sensibilidade e VPN foram encontrados nesses índices. Nessa análise, novamente os IC95\% indicaram similaridade no desempenho dos quatro índices. Para mortalidade hospitalar nesse grupo, o SAPS III apresentou maior valor de AUC $(0,818)$, sem diferir significativamente dos demais, uma vez que os IC95\% se sobrepõem (Tabela 2).

Tabela 2 - Capacidade preditiva do RTS, REMS, mREMS e SAPS III para mortalidade em UTI e hospitalar de vítimas de trauma contuso em tratamento cirúrgico - São Paulo, SP, Brasil, 2014-2016.

\begin{tabular}{lcccc}
\hline \multirow{2}{*}{ Métricas da ROC } & \multicolumn{2}{c}{ Mortalidade em UTI } \\
\cline { 2 - 5 } AUC/ROC & RTS & REMS & mREMS & 0,811 \\
IC95\% & 0,747 & 0,753 & 0,802 & $0,726-0,896$ \\
Ponto de corte & $0,627-0,868$ & $0,636-0,870$ & $0,697-0,907$ & 44,5 \\
Sensibilidade & 6,2 & 5,5 & 4,5 & $60,7 \%$ \\
Especificidade & $80,0 \%$ & $82,0 \%$ & $65,2 \%$ & $95,0 \%$ \\
VPP & $68,5 \%$ & $65,0 \%$ & $90,0 \%$ & $98,2 \%$ \\
VPN & $36,4 \%$ & $91,2 \%$ & $35,2 \%$ \\
\hline Métricas da ROC & $93,8 \%$ & $44,8 \%$ & $36,7 \%$ & SAPS III \\
\hline AUC/ROC & \multicolumn{2}{c}{ Mortalidade hospitalar } & mREMS \\
IC95\% & RTS & REMS & 0,818 \\
Ponto de corte & 0,703 & 0,706 & 0,737 & $0,736-0,900$ \\
Sensibilidade & $0,584-0,822$ & $0,589-0,824$ & $0,622-0,852$ & 44,5 \\
\hline
\end{tabular}


...continuação

\begin{tabular}{lcccc}
\hline \multirow{2}{*}{ Métricas da ROC } & \multicolumn{3}{c}{ Mortalidade hospitalar } \\
\cline { 2 - 5 } & RTS & REMS & mREMS & SAPS III \\
\hline Especificidade & $69,0 \%$ & $56,0 \%$ & $56,0 \%$ & $92,0 \%$ \\
VPP & $40,9 \%$ & $86,2 \%$ & $86,6 \%$ & $96,4 \%$ \\
VPN & $89,2 \%$ & $48,3 \%$ & $51,8 \%$ & $42,6 \%$ \\
\hline
\end{tabular}

AUC/ROC - Area Under Curve/Receiver Operating Characteristic; RTS - Revised Trauma Score; REMS - Rapid Emergency Medicine Score; mREMS - modified Rapid Emergency Medicine Score; SAPS III - Simplified Acute Physiology Score III; UTI - Unidade de Terapia Intensiva; IC - Intervalo de Confiança; VPP - Valor Preditivo Positivo; VPN - Valor Preditivo Negativo $(\mathrm{n}=110)$.

A Figura 3 mostra resultados que indicam desempenho insatisfatório dos índices para estimar a mortalidade em UTI de vítimas de trauma contuso sem tratamento cirúrgico. Todos os índices apresentam AUC bem próximas a 0,50:
0,568 (IC95\%: 0,333-0,803) para o RTS; 0,528 (IC95\%: 0,307-0,749) para o REMS; 0,612 (IC95\%: 0,402-0,821) para o mREMS e 0,582 (IC95\%: 0,373-0,792) para o SAPS III.

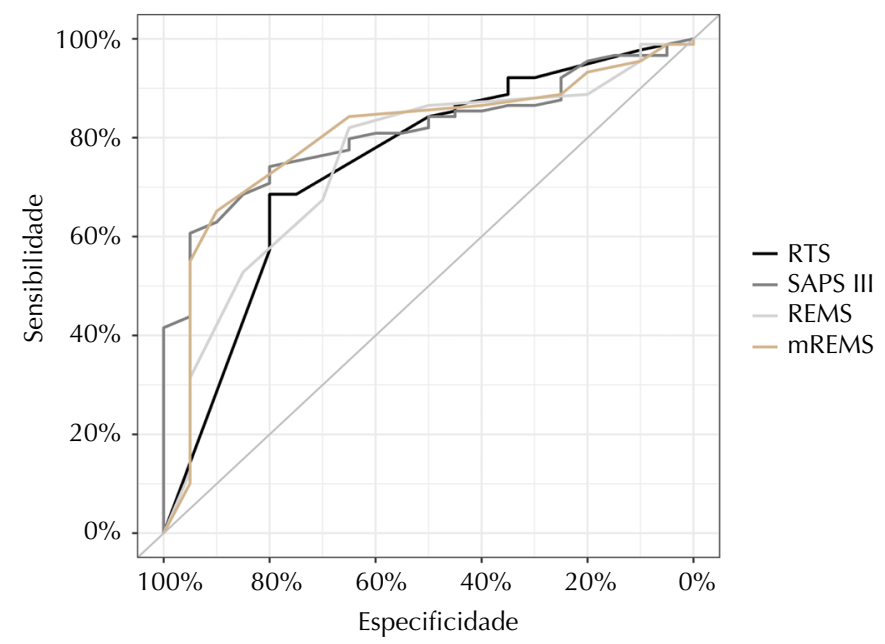

ROC - Receiver Operating Characteristic; RTS - Revised Trauma Score; SAPS III - Simplified Acute Physiology Score III; REMS - Rapid Emergency Medicine Score; mREMS - modified Rapid Emergency Medicine Score; UTI - Unidade de Terapia Intensiva ( $n=110)$.

Figura 2 - Curvas ROC dos índices RTS, SAPS III, REMS e mREMS para mortalidade em UTI de vítimas de trauma submetidas a cirurgia - São Paulo, SP, Brasil, 2014-2016.

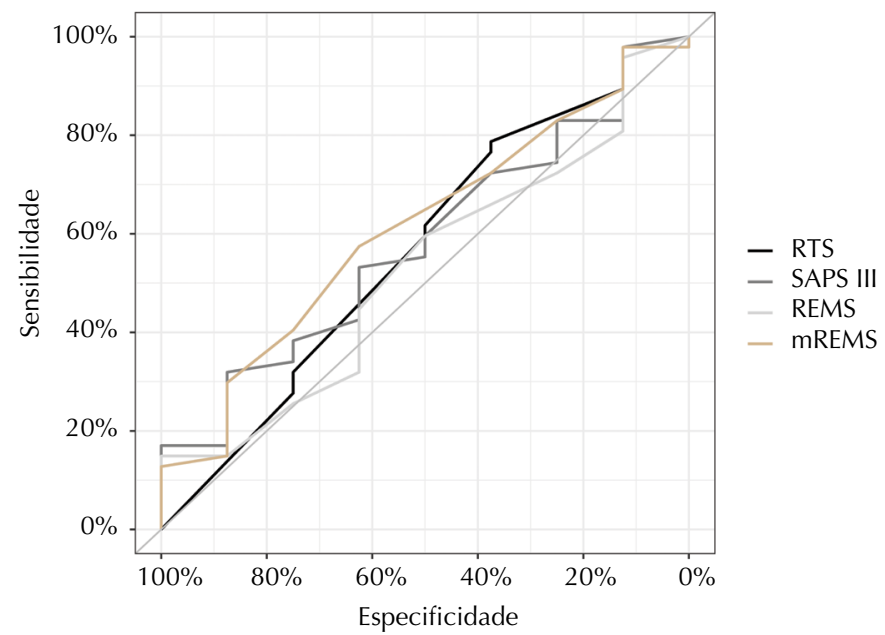

ROC - Receiver Operating Characteristic; RTS - Revised Trauma Score; SAPS III - Simplified Acute Physiology Score III; REMS - Rapid Emergency Medicine Score; mREMS - modified Rapid Emergency Medicine Score; UTI - Unidade de Terapia Intensiva ( $\mathrm{n}=55$ ).

Figura 3 - Curvas ROC dos índices RTS, SAPS III, REMS e mREMS para mortalidade em UTI de vítimas de trauma sem tratamento cirúrgico - São Paulo, SP, Brasil, 2014-2016. 
Valores idênticos foram observados para mortalidade hospitalar. Nenhuma vítima de trauma contuso sem tratamento cirúrgico morreu após alta da UTI; portanto, foi equivalente o desempenho insatisfatório dos índices para estimar mortalidade na UTI e hospital nesses pacientes.

\section{DISCUSSÃO}

Para analisar o comportamento dos índices em relação à capacidade de discriminar mortos e sobreviventes na UTI e no hospital, aplicou-se a curva ROC, considerando prioritariamente a AUC/ROC, tendo em vista sua habilidade de indicar a capacidade prognóstica global dos instrumentos. A análise do resultado dessa estatística na casuística total da investigação mostrou desempenho razoável dos instrumentos para prática clínica, com capacidade prognóstica mais satisfatória do mREMS e SAPS III na UTI e do SAPS III na previsão de mortalidade hospitalar (AUC/ROC superiores a 0,70$)^{(12)}$, embora essa diferença não tenha alcançado o nível de significância estatística estabelecido neste estudo.

Há de se considerar também que, no ponto de corte estabelecido pelo índice de Youden, o SAPS III apresentou a pior sensibilidade e VPN em relação aos demais instrumentos, identificando aproximadamente metade dos mortos da casuística: 49,3\% dos óbitos ocorridos na UTI e 50,4\% das mortes hospitalares (valores da sensibilidade); ademais, quando o SAPS III indicou sobrevida, ele prognosticou corretamente somente $27,4 \%$ dos casos na UTI e 31,6\% no hospital (VPN).

Por outro lado, o SAPS III apresentou alta especificidade e VPP; logo, foi um bom índice para identificar sobrevivência dentro da casuística (indicou 92,9\% e 90,9\% dos que sobreviveram na UTI e hospital, respectivamente) e quando indicou morte, prognosticou corretamente a maioria dos casos $(97,1 \%$ na UTI e $95,6 \%$ no hospital).

Diferentemente dos demais índices, o RTS apresentou valores mais elevados de sensibilidade e VPN do que especificidade e VPP; entretanto, na interpretação desses resultados, é essencial considerar que as mais altas pontuações nesse índice apontam maior probabilidade de sobrevida, ao passo que nos demais instrumentos maiores escores indicam morte.

Tendo em vista esse aspecto, o RTS e o SAPS III tiveram maior capacidade de identificar os sobreviventes do que os mortos na casuística, enquanto o REMS identificou de forma similar esses desfechos e o mREMS discriminou melhor os indivíduos que morreram no hospital do que na UTI (sensibilidade de 74,8\% e 52,9\%, respectivamente).

Os índices de gravidade são instrumentos elaborados para a prática clínica visando avaliar a qualidade da assistência prestada aos pacientes e o planejamento de cuidados emergenciais ${ }^{(13-14)}$. Nesse sentido, é de especial interesse sua capacidade de discriminar corretamente indivíduos com alta probabilidade de morrer ou viver. Em consequência, a AUC/ROC que quantificou a capacidade geral dos índices para realizar essa discriminação e identificar corretamente mortos e vivos trouxe a síntese dos resultados de interesse para esta investigação.

Segundo a AUC/ROC, vítimas submetidas a tratamento cirúrgico tiveram predições de mortalidade com melhor desempenho em UTI do que as que não receberam esse tipo de tratamento - variação da AUC/ROC entre 0,747 e 0,811 para casos cirúrgicos e entre 0,528 e 0,612 para os demais.
Nos casos cirúrgicos, o SAPS III e o mREMS alcançaram boa capacidade discriminatória com AUC/ROC superior a 0,80. Em contrapartida, nos casos não cirúrgicos, os índices tiveram desempenho insatisfatório (AUC/ROC < 0,70); além disso, na Figura 3, observa-se que a curva do REMS foi superada em alguns segmentos pela referência da curva ROC. Essa linha diagonal do gráfico representa o comportamento dos índices se os escores não produzem informação alguma sobre o prognóstico das vítimas. Portanto, o traçado do REMS sugere que em algumas pontuações o índice não teve capacidade discriminatória para mortalidade em UTI em vítimas de trauma não submetidas a tratamento cirúrgico.

Quanto à morte hospitalar em vítimas com tratamento cirúrgico, resultados semelhantes aos relacionados à mortalidade em UTI foram encontrados e, novamente, houve melhora dos valores da AUC/ROC quando pacientes cirúrgicos foram analisados.

Os valores da AUC/ROC apresentados nos artigos originais dos índices selecionados para esta investigação foram: 0,852 (dp = 0,014) para o $\mathrm{REMS}^{(9)}, 0,967$ (IC95\%: 0,9630,971) para o mREMS ${ }^{(10)}$ e 0,83 para o SAPS III ${ }^{(11)}$. O RTS triagem, que considerava os valores da ECGl, PAS e FR das vítimas na cena do evento traumático, identificou mais de 97\% dos não sobreviventes na investigação apresentada na sua primeira publicação, no Journal of Trauma em $1989^{(8)}$.

$\mathrm{Na}$ atual pesquisa, a AUC/ROC entre os índices foi similar quando analisada mortalidade em UTI e hospitalar na casuística total e quando investigados, separadamente, pacientes submetidos ou não a cirurgia. Portanto, a capacidade prognóstica do RTS, REMS, mREMS e SAPS III foi equivalente. $\mathrm{Na}$ revisão de literatura, não foram observados artigos que compararam o SAPS III com os demais índices analisados na atual investigação. Em relação ao mREMS, a comparação entre índices somente foi encontrada na publicação do artigo original, comentada na introdução deste estudo.

O REMS foi confrontado ao RTS em três estudos, em que outros índices também foram incluídos nas comparações ${ }^{(15-17)}$. Nesses estudos, o REMS e RTS tiveram desempenho semelhante; em duas dessas investigações, os índices apresentaram excelente capacidade prognóstica, AUC/ROC de 0,91 e 0,9 para o REMS e 0,89 e 0,924 para o $\operatorname{RTS}^{(15,17)}$. Valores aceitáveis, de 0,72 e 0,77 de AUC/ROC, foram observados em outra pesquisa ${ }^{(16)}$.

O SAPS III é um índice de gravidade da doença em UTI; em consequência, não foi observada na revisão de literatura sua aplicação em vítimas de trauma, embora estudos tenham aplicado sua versão anterior, SAPS II, nessa população. Todavia, encontraram-se valores da AUC/ROC desse índice referente a diversas situações clínicas. São elas: pacientes internados em UTI geral (AUC 0,73) e (AUC 0,901)(18-19); transplantes de órgãos em geral (AUC 0,696) (20); transplantes hepáticos (AUC 0,612) ${ }^{(20)}$; transplantes renais (AUC 0,459) ${ }^{(20)}$; transplantes pulmonares (AUC 0,792) ${ }^{(21)}$; pacientes internados em unidade coronariana $(\text { AUC 0,84) })^{(21)}$; após parada cardiorrespiratória (AUC 0,621) e (AUC 0,74)(22-23); pacientes com câncer (AUC 0,948)(24) e choque séptico (AUC 0,817) ${ }^{(25)}$. A variação do valor da AUC/ROC foi de 0,459 a 0,948 . A pior acurácia foi observada em transplantes renais $^{(20)}$ e a melhor, em pacientes com câncer ${ }^{(24)}$.

Nos estudos que compararam o SAPS III com outros índices, foram frequentes as análises com o SAPS II e 
APACHE II. No geral, o SAPS II apresentou melhor desempenho do que o SAPS III quando comparadas AUC/ ROC e o SAPS III superou o APACHE (18-20,24-26). $^{\text {. }}$

Diante da alta mortalidade hospitalar identificada neste estudo (20,6\%), foi pequena a frequência de casos do REMS e mREMS com pontuação > 13 (inferior a $2 \%$ ), ponto de corte dos índices considerado como alerta de alto risco de morrer ${ }^{(9-10)}$. Portanto, as pontuações desses índices não refletiram a gravidade da casuística analisada.

Resultados mais ajustados à mortalidade observada foram encontrados no RTS e SAPS III. Em relação ao RTS, $18,7 \%$ da casuística apresentaram estimativa de probabilidade de sobrevida igual ou inferior a $50 \%$, tendo, portanto, alta probabilidade de morte. A mortalidade observada na UTI, de 17,6\%, e o valor médio do SAPS III das vítimas também foram convergentes, quando se observa a pontuação média do índice que indica $15,9 \%$ de probabilidade de morte em UTI quando convertida por equação de regressão.

Esta pesquisa apresenta como limitações a busca de dados em prontuários e a realização do estudo com informações de uma única UTI, especializada no atendimento de vítimas de trauma e localizada em hospital que atende somente pacientes referenciados em seu departamento de emergência. A ausência de um banco de registros sistematizados, contendo dados relevantes das vítimas de trauma, como se observa em países desenvolvidos, tornou difícil e menos segura a realização deste estudo.
As características do local de estudo certamente trouxeram especificidades à casuística analisada; nesse sentido, vale destacar que $53,4 \%$ dos participantes chegaram ao departamento de emergência trazidos por unidades avançadas aéreas ou terrestres. Esse percentual elevado de atendimento pré-hospitalar avançado indica a identificação de condições clínicas graves na maioria das vítimas já na cena da ocorrência. Além do mais, a presença de enfermeiros e médicos nessas unidades possibilitou a realização precoce de procedimentos invasivos e complexos que influenciam no tempo de sobrevivência das vítimas, tais como procedimentos respiratórios avançados e uso de medicamentos relacionados à reanimação cardiopulmonar ${ }^{(27)}$. Esses procedimentos, quando iniciados ainda no atendimento pré-hospitalar, podem estabilizar as condições circulatórias e ventilatórias das vítimas e atenuar a gravidade indicada pelo RTS, REMS e mREMS, que utilizam para seu cálculo os sinais vitais iniciais mensurados no departamento de emergência.

\section{CONCLUSÃO}

A capacidade prognóstica do RTS, REMS, mREMS e SAPS III foi moderada e similar, não havendo indicação preferencial de um desses escores para uso na prática clínica. Além disso, o melhor desempenho alcançado nos índices, quando aplicados em pacientes cirúrgicos, traz a proposição de que esse grupo de vítimas será mais beneficiado do que os demais pacientes com o uso desses indicadores.

\section{RESUMO}

Objetivo: Identificar a capacidade preditiva de mortalidade dos índices Revised Trauma Score, Rapid Emergency Medicine Score, modified Rapid Emergency Medicine Score e Simplified Acute Physiology Score III em vítimas de trauma contuso internadas em unidade de terapia intensiva e comparar seu desempenho. Método: Coorte retrospectiva de pacientes com trauma contuso de uma unidade de terapia intensiva a partir do registro em prontuários. Receiver Operating Characteristic e intervalo de confiança de $95 \%$ da área sob a curva foram analisados para comparar os resultados. Resultados: Dos 165 pacientes analisados, $66,7 \%$ tiveram tratamento cirúrgico. A mortalidade na unidade de terapia intensiva e no hospital foi de $17,6 \%$ e 20,6\%, respectivamente. Para mortalidade na terapia intensiva, houve variação das áreas sob a curva entre 0,672 e 0,738; porém, melhores resultados foram observados em pacientes cirúrgicos (0,747 a 0,811). Resultados similares foram observados para mortalidade hospitalar. Em todas as análises, as áreas sob a curva dos índices não diferiram significativamente. Conclusão: Houve acurácia moderada dos índices de gravidade, com melhora na performance quando aplicados em pacientes cirúrgicos. Os quatro índices apresentaram predição similar para os desfechos analisados.

\section{DESCRITORES}

Ferimentos e Lesões; Índice de Gravidade da Doença; Índices de Gravidade do Trauma; Mortalidade; Prognóstico; Curva ROC.

\section{RESUMEN}

Objetivo: Identificar la capacidad predictiva de la mortalidad de los índices Revised Trauma Score, Rapid Emergency Medicine Score, modified Rapid Emergency Medicine Score y Simplified Acute Physiology Score III en víctimas de traumas contusos hospitalizadas en una unidad de cuidados intensivos y comparar su rendimiento. Método: Cohorte retrospectiva de pacientes con trauma contuso en una unidad de cuidados intensivos a partir de los registros médicos. Se analizaron el Receiver Operating Characteristic y el intervalo de confianza del $95 \%$ del área bajo la curva para comparar los resultados. Resultados: De los 165 pacientes analizados, el 66,7\% recibió tratamiento quirúrgico. La mortalidad en la unidad de cuidados intensivos y en el hospital fue del 17,6\% y del 20,6\%, respectivamente. Para la mortalidad en la unidad de cuidados intensivos, las áreas bajo la curva oscilaron entre 0,672 y 0,738; sin embargo, se observaron mejores resultados en los pacientes quirúrgicos $(0,747$ a 0,811$)$. Se observaron resultados similares para la mortalidad hospitalaria. En todos los análisis, las áreas bajo la curva de los índices no difieren significativamente. Conclusión: La precisión de los índices de gravedad fue moderada, con un mejor rendimiento cuando se aplicaron a pacientes quirúrgicos. Los cuatro índices mostraron una predicción similar para los resultados analizados.

\section{DESCRIPTORES}

Heridas y Traumatismos; Índice de la Severidad de la Enfermedad; Índices de Gravidad del Trauma; Mortalidad; Prognóstico; Curva ROC.

\section{REFERÊNCIAS}

1. Brasil. Ministério da Saúde. DATASUS. Informações e saúde [Internet]. Brasília, DF; 2020 [cited 2020 Jan 20]. Available from: http:// datasus.saude.gov.br/.

2. Instituto Brasileiro de Geografia e Estatística. Sistema IBGE de Recuperação Automática - SIDRA. Estimativas de população [Internet]. Rio de Janeiro: Instituto Brasileiro de Geografia e Estatística; 2021 [cited 2021 Apr 26]. Available from: https://sidra.ibge.gov.br/tabela/6579\# resultado 
3. Christensen MC, Banner C, Lefering R, Vallejo-Torres L, Morris S. Quality of life after severe trauma: results from the global trauma trial with recombinant Factor VII. J Trauma. 2011;70(6):1524-31. https://doi.org/10.1097/TA.0b013e3181f053c2

4. Silva LC, Nogueira LS, Settervall CH, Sousa RM, Padilha KG. [The use of severity indexes to estimate the risk of death in Intensive Care]. Rev Esc Enferm USP. 2012;46(4):846-50. Portuguese. https://doi.org/10.1590/S0080-62342012000400009

5. Nogueira LS, Sousa RM, Domingues CA. Severity of trauma victims admitted in intensive care units: comparative study among different indexes. Rev Lat Am Enfermagem. 2009;17(6):1037-42. https://doi.org/10.1590/S0104-11692009000600017

6. Keegan MT, Soares M. What every intensivist should know about prognostic scoring systems and risk-adjusted mortality. Rev Bras Ter Intensiva. 2016;28(3):264-9. https://doi.org/10.5935/0103-507X.20160052

7. Lefering R. Trauma scoring systems. Curr Opin Crit Care. 2012;18(6):637-40. https://doi.org/10.1097/MCC.0b013e3283585356

8. Champion HR, Sacco WJ, Copes WS, Gann DS, Gennarelli TA, Flanagan ME. A revision of the trauma score. J Trauma. 1989;29(5):623-9. https://doi.org/10.1097/00005373-198905000-00017

9. Olsson T, Terent A, Lind L. Rapid emergency medicine score: a new prognostic tool for in-hospital mortality in nonsurgical emergency department patients. J Intern Med. 2004;255(5):579-87. https://doi.org/10.1111/j.1365-2796.2004.01321.x

10. Miller RT, Nazir N, McDonald T, Cannon CM. The modified rapid emergency medicine score: a novel trauma triage tool to predict inhospital mortality. Injury. 2017;48(9):1870-7. https://doi.org/10.1016/j.injury.2017.04.048

11. Moreno RP, Metnitz PG, Almeida E, Jordan B, Bauer P, Campos RA, et al. SAPS 3-From evaluation of the patient to evaluation of the intensive care unit. Part 2: development of a prognostic model for hospital mortality at ICU admission. Intensive Care Med. 2005;31(10):1345-55. https://doi.org/10.1007/s00134-005-2763-5

12. Carter JV, Pan J, Rai SN, Galandiuk S. ROC-ing along: evaluation and interpretation of receiver operating characteristic curves. Surgery. 2016;159(6):1638-45. https://doi.org/10.1016/j.surg.2015.12.029

13. Ali Ali B, Fortún Moral M, Belzunegui Otano T, Reyero Díez D, Castro Neira M. Escalas para predicción de resultados tras traumatismo grave. An Sist Sanit Navar. 2017;40(1):103-18. https://doi.org/10.23938/assn.0001

14. Maas AI, Menon DK, Adelson PD, Andelic N, Bell MJ, Belli A, et al. Traumatic brain injury: integrated approaches to improve prevention, clinical care, and research. Lancet Neurol. 2017;16(12):987-1048. https://doi.org/10.1016/S1474-4422(17)30371-X

15. Park HO, Kim JW, Kim SH, Moon SH, Byun JH, Kim KN, et al. Usability verification of the Emergency Trauma Score (EMTRAS) and Rapid Emergency Medicine Score (REMS) in patients with trauma: a retrospective cohort study. Medicine (Baltimore). 2017;96(44):e8449. https:// doi.org/10.1097/MD.0000000000008449

16. Moore L, Lavoie A, LeSage N, Abdous B, Bergeron E, Liberman M, et al. Statistical validation of the Revised Trauma Score. J Trauma. 2006;60(2):305-11. https://doi.org/10.1097/01.ta.0000200840.89685.b0

17. Imhoff BF, Thompson NJ, Hastings MA, Nazir N, Moncure M, Cannon CM. Rapid Emergency Medicine Score (REMS) in the trauma population: a retrospective study. BMJ Open. 2014;4(5):e004738. https://doi.org/10.1136/bmjopen-2013-004738

18. Juneja D, Singh O, Nasa P, Dang R. Comparison of newer scoring systems with the conventional scoring systems in general intensive care population. Minerva Anestesiol. 2012;78(2):194-200.

19. Katsounas A, Kamacharova L, Tyczynski B, Eggebrecht H, Erbel R, Cambay A, et al. The predictive performance of the SAPS II and SAPS 3 scoring systems: a retrospective analysis. J Crit Care. 2016;33:180-5. https://doi.org/10.1016/j.jcrc.2016.01.013

20. Oliveira VM, Brauner JS, Rodrigues Filho E, Susin RG, Draghetti V, Bolzan ST, et al. Is SAPS 3 better than APACHE II at predicting mortality in critically ill transplant patients? Clinics (São Paulo). 2013;68(2):153-8. https://doi.org/10.6061/clinics/2013(02)OA06

21. Nassar AP, Mocelin AO, Andrade FM, Brauer L, Gianini FP, Nunes AL, et al. SAPS 3, APACHE IV or GRACE: which score to choose for acute coronary syndrome patients in intensive care units? Sao Paulo Med J. 2013;131(3):173-8 173. https://doi.org/10.1590/1516-3180.2013.1313474

22. Salciccioli JD, Cristia C, Chase M, Giberson T, Graver A, Gautam S, et al. Performance of SAPS II and SAPS III scores in post-cardiac arrest. Minerva Anestesiol [Internet]. 2012;78(12):1341-7. Available from: https://pubmed.ncbi.nlm.nih.gov/22743785/

23. Bisbal M, Jouve E, Papazian L, de Bourmont S, Perrin G, Eon B, et al. Effectiveness of SAPS III to predict hospital mortality for post-cardiac arrest patients. Resuscitation. 2014;85(7):939-44. https://doi.org/10.1016/j.resuscitation.2014.03.302

24. Xing X, Gao Y, Wang H, Huang C, Qu S, Zhang H, et al. Performance of three prognostic models in patients with cancer in need of intensive care in a medical center in China. PLoS One. 2015;10(6):e0131329. https://doi.org/10.1371/journal.pone.0131329

25. Khwannimit B, Bhurayanontachai R, Vattanavanit V. Validation of the Sepsis Severity Score Compared with Updated Severity Scores in Predicting Hospital Mortality in Sepsis Patients. Shock. 2017;47(6):720-5. https://doi.org/10.1097/SHK.0000000000000818

26. Ho KM, Williams TA, Harahsheh Y, Higgins TL. Using patient admission characteristics alone to predict mortality of critically ill patients: a comparison of 3 prognostic scores. J Crit Care. 2016;31(1):21-5. https://doi.org/10.1016/j.jcrc.2015.10.019

27. Schweitzer G, Nascimento ER, Nascimento KC, Moreira AR, Amante LN, Malfussi LB. Emergency interventions for air medical services trauma victims. Rev Bras Enferm. 2017;70(1):54-60. https://doi.org/10.1590/0034-7167-2016-0311

\section{Apoio financeiro}

Coordenação de Aperfeiçoamento de Pessoal de Nível Superior (Capes), Código de Financiamento 001. Conselho Nacional de Desenvolvimento Científico e Tecnológico (CNPq), Processo no 148766/2016-1. 